Revue des patrimoines

25 | 2014

Le patrimoine de la Grande Guerre

\title{
Le Monument à la Victoire et à la Paix de Paul Landowski. Une découverte récente à Casablanca
}

Nicolas Alexandre and Emmanuel Neiger

\section{(2) OpenEdition}

1 Journals

Electronic version

URL: http://journals.openedition.org/insitu/11360

DOI: 10.4000/insitu. 11360

ISSN: 1630-7305

Publisher

Ministère de la Culture

Electronic reference

Nicolas Alexandre and Emmanuel Neiger, «Le Monument à la Victoire et à la Paix de Paul Landowski. Une découverte récente à Casablanca », In Situ [Online], 25 | 2014, Online since 09 December 2014, connection on 25 June 2020. URL : http://journals.openedition.org/insitu/11360 ; DOI : https:// doi.org/10.4000/insitu. 11360

This text was automatically generated on 25 June 2020.

\section{cc) $(1) \odot$}

In Situ Revues des patrimoines est mis à disposition selon les termes de la licence Creative Commons Attribution - Pas d'Utilisation Commerciale - Pas de Modification 4.0 International. 


\title{
Le Monument à la Victoire et à la Paix de Paul Landowski. Une découverte récente à Casablanca
}

\author{
Nicolas Alexandre and Emmanuel Neiger
}

Nous tenons à exprimer notre profonde gratitude à Bernard Toulier, conservateur général honoraire du patrimoine, qui était présent avec nous lors de la découverte des plâtres, pour son soutien et ses conseils avisés. Les remarques pertinentes de Pascal Plaza méritent ici notre reconnaissance. Les compétences linguistiques de Pascale Abdelkhirane nous ont également été précieuses pour la traduction anglaise.

\section{Le Maroc et la Première Guerre mondiale}

Alors que le protectorat français est établi au Maroc depuis seulement deux ans avec le traité de Fès en 1912, la Première Guerre mondiale éclate et le général Lyautey, premier résident général, ne peut soustraire ce nouveau territoire aux obligations de l'effort de guerre : il est ainsi contraint d'envoyer une grande partie des troupes dont il dispose et même des troupes «indigènes ». Les réticences sont pourtant nombreuses: Lyautey veut poursuivre sa politique de "pacification » vers l'intérieur des terres du Maroc et asseoir l'organisation de "son" protectorat, de même qu'il veut en assurer l'essor industriel et commercial malgré le conflit; par ailleurs, engager des troupes marocaines aux côtés des troupes françaises s'avère délicat dans la mesure où la résistance à l'arrivée de la France est vive, ce qui fait craindre des désertions ou des exactions. Malgré tout, sont envoyées en France des troupes composées de Français, Tunisiens, Algériens et Sénégalais qui étaient postées à Casablanca ainsi que nombre de Marocains ${ }^{1}$. 


\section{Un monument en contexte : la place Mohamed V}

Après l'armistice et pendant les années 20, la ville de Casablanca va connaître un essor inédit: avec la présence de l'urbaniste du Musée social Henri Prost, un plan d'urbanisme est établi ${ }^{2}$ qui dessine au centre de la nouvelle ville une place civique, la place administrative qui deviendra un temps la place Lyautey avant d'être renommée, après l'indépendance du pays, la place Mohamed $\mathrm{V}$.

Figure 1

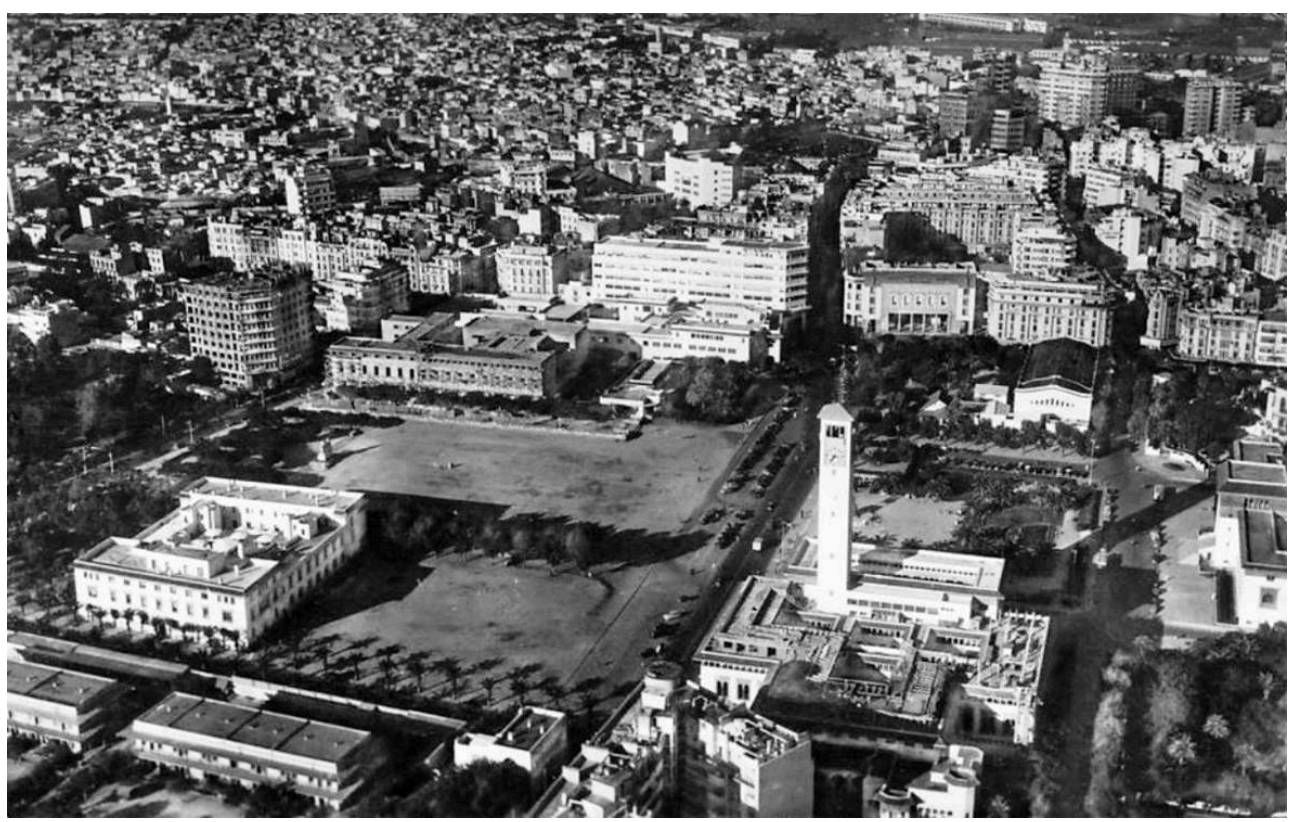

Place mohamed v (eX. Place lyautey), début des anNées 1950. Carte postale, Flandrin éd. Collection particulière.

Repro. Alexandre, Nicolas. () Nicolas Alexandre.

Or, cette place à l'ordonnance classique (fig. 1), que Lyautey veut dans l'esprit de la place Stanislas à $\mathrm{Nancy}^{3}$, doit constituer le centre du pouvoir européen dans une ville essentiellement nouvelle et industrielle ; il s'agira donc d'y implanter les symboles du pouvoir judiciaire avec l'édification du tribunal de grande instance qui surplombe la place, militaire, avec deux bâtiments, en retrait cependant, le cercle des officiers et la subdivision militaire et administratif avec la trésorerie générale et plus tard la construction de l'hôtel de ville. L'identité industrielle et commerciale de la ville s'y lit également avec l'édification de la Banque d'État. Cette place joue aussi un rôle social et culturel: on y trouve la grande poste, le théâtre, le siège de l'Automobile Club. S'ajoutent à ces édifices deux monuments symboliques : le monument de la Victoire de Paul Landowski qui nous occupe, puis, la statue du maréchal Lyautey inaugurée en 1938 et réalisée par le sculpteur François Cogné ${ }^{4}$. Le choix de Casablanca, plutôt que Rabat, siège de la Résidence, peut s'expliquer de deux façons. Tout d'abord, les deux monuments, s'ils s'adressent à toute la population, se situent sur ce lieu du nouveau pouvoir, dans la ville où la communauté française est la plus nombreuse ${ }^{5}$. Ce n'est bien entendu pas fortuit. C'est un signal fort pour cette communauté. De plus, le port casablancais était aussi le lieu de départ des troupes mobilisées pour le front européen. La présence d'un monument du souvenir commémorant l'engagement de troupes 
françaises et marocaines en provenance du Maroc trouvait naturellement sa place au cœur de cette ville. Dans ce lieu, s'affichent d'ailleurs d'autres signes de l'établissement historique du protectorat, avec particulièrement la réplique de la cabane du général Drude, l'officier qui a pris la ville de Casablanca en août 1907, accompagnée d'une plaque commémorative du débarquement français. Ce sera également le théâtre de toutes les manifestations officielles organisées par l'administration française.

\section{Un monument de l'entraide franco-marocaine}

L'emplacement réservé au Monument à la Victoire et à la Paix, au centre nord-ouest de la place, lui donne une portée symbolique forte : il s'agit de commémorer l'entraide entre le Maroc et la France lors de la Grande Guerre.

Le monument est commandité par l'Association casablancaise des vétérans qui a fait appel à une souscription.

Figure 2

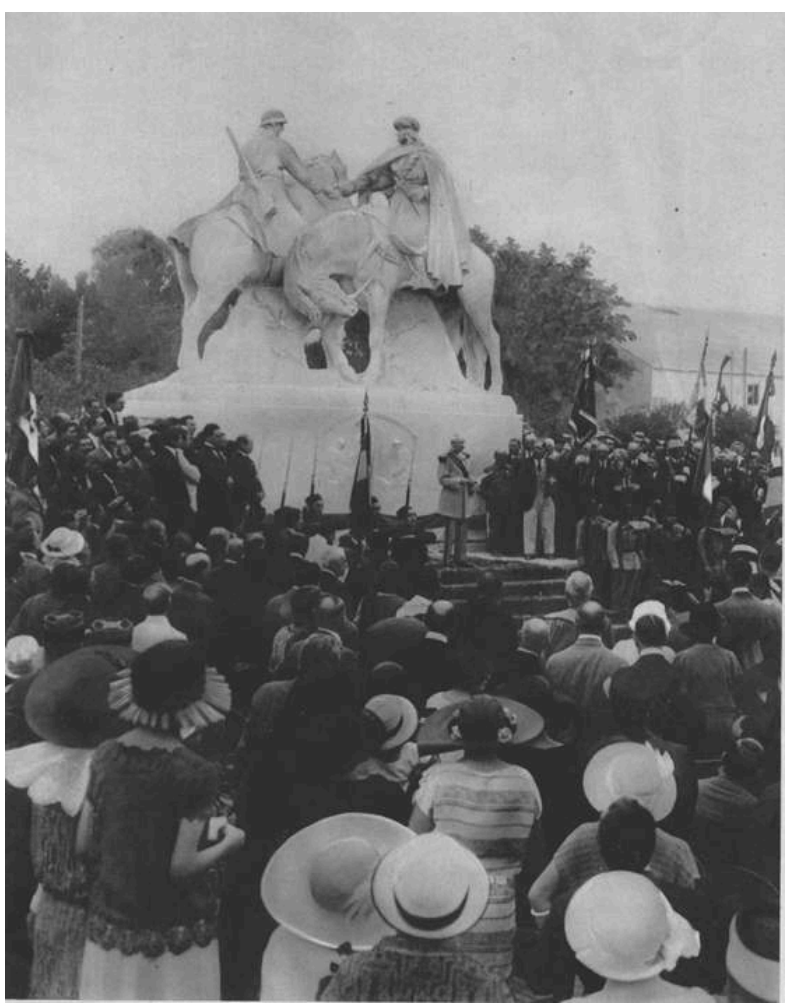

INAUgURATION DU MONUMENT À LA VICTOIRE ET À LA PAIX, 20 JUILLET 1924. PHOT. FLANDRIN ÉD. COLLECTION PARTICULIÈRE.

REPRO. ALEXANDRE, NICOLAS. @ NICOLAS ALEXANDRE.

Au moment de son inauguration, le 20 juillet $1924^{6}$ (fig. 2), en pleine guerre du Rif, un « délégué des associations de blessés, mutilés, victimes de la Grande Guerre, anciens combattants domiciliés au Maroc », Me Rolland, revendique les droits de "ceux dont nous sommes les protecteurs et les tuteurs naturels » et affirme à propos des soldats marocains :

Vous savez que le statut des mutilés marocains a toujours été la première de nos revendications. Moins bien placés que nous pour se défendre et pour faire entendre 
leurs doléances, nos camarades indigènes nous semblent avoir, plus encore que nous, droit à votre bienveillante attention ${ }^{7}$.

Ces propos témoignent de l'esprit somme toute paternaliste de l'époque, à la fois bien intentionné, dans la volonté de reconnaître aux combattants marocains les droits que la loi française accordait aux combattants de nationalité française en métropole, et colonialiste dans l'affirmation d'une hiérarchie naturelle entre les peuples.

De son côté, lors de la même cérémonie, le maréchal Lyautey affirme :

Le président de l'association des combattants, le pacha de Casablanca, le président général des Vétérans viennent d'interpréter éloquemment les sentiments qui nous animent tous au pied de ce monument. Ce que je retiens avant tout de leurs paroles, c'est ce que symbolisent si hautement ces deux guerriers aux mains unies, l'UNION DES DEUX RACES qu'une suite d'événements historiques a appelés à vivre côte à côte sur cette terre marocaine [...] Et ce qui me touche peut-être le plus dans les paroles que je viens d'entendre, c'est que ce soit d'une bouche française, la vôtre Monsieur le Président des anciens combattants, que sorte cette chaleureuse revendication en faveur des camarades musulmans dont un oubli législatif avait si injustement méconnu les titres. Vous avez bien voulu rappeler mes efforts pour obtenir la réparation d'une illégalité injustifiable.

Il ajoute :

Tous, Français et Marocains, formant les légendaires Divisions marocaines, sont allés ensemble vers la Mort, mais aussi vers la Gloire et vers la Victoire. Tant d'entre eux dorment aujourd'hui côte à côte sur les champs de France, au pied des tranchées où ils ont tenu jusqu'au dernier souffle, et c'est eux dont nous célébrons aujourd'hui pieusement la mémoire devant ce monument élevé aux Morts, à la Gloire à la Victoire et à la Paix ${ }^{8}$.

Lyautey souligne ainsi, d'un ton volontiers lyrique, l'enjeu somme toute politique de l'édification de ce monument: il ne s'agit pas seulement de rendre hommage aux soldats marocains morts pour la France, mais aussi de consolider l'amitié francomarocaine au moment même où la France affronte aux côtés de l'Espagne les troupes d'Abd-el-Krim dans le Rif et où le résident général cherche à prouver que le protectorat au Maroc n'a rien à voir avec le principe de la colonisation établie en Algérie ${ }^{9}$. Lyautey désire en effet appuyer sa gouvernance sur la conservation du pouvoir central makhzénien $^{10}$ et de l'aristocratie locale. Mettre en valeur cette entraide, par un monument placé au centre de la ville, revient donc à signifier que le protectorat constitue une collaboration ${ }^{11}$.

Cependant, la présence des autorités marocaines à la cérémonie se résume à celle du pacha de Casablanca ${ }^{12}$, Si Abdelouahad, qui pose à côté du Maréchal Lyautey pour les photographies et dont le discours se résume à des propos convenus et diplomatiques, et à celle du grand vizir El Mokri. La présence de la population marocaine quant à elle pose problème : une seule photographie sur celles que nous avons pu consulter montre la présence de Marocains en habits traditionnels ${ }^{13}$, qui plus est en couverture de la revue alors que la photographie la plus nette (voir fig. 2) laisse voir nettement que le maréchal Lyautey n'est entouré que d'Européens.

\section{Une œuvre de Paul Landowski}

Le monument est réalisé par le célèbre sculpteur Paul Landowski (1875-1961) (fig. 3). Entré à l'École des beaux-arts en 1895, il obtient un Premier Grand Prix de Rome en 1900 pour un David combattant qui lui ouvre les portes de la villa Médicis pour quatre 
années. C'est le commencement d'une carrière classique et académique qui lui donne accès à une célébrité précoce. Son œuvre a été marquée par une production largement alimentée par une commande privée et publique avec des œuvres de rayonnement international comme le Mur des Réformateurs de Genève (1909-1917) ou le Christ rédempteur de Rio (1931).

Figure 3

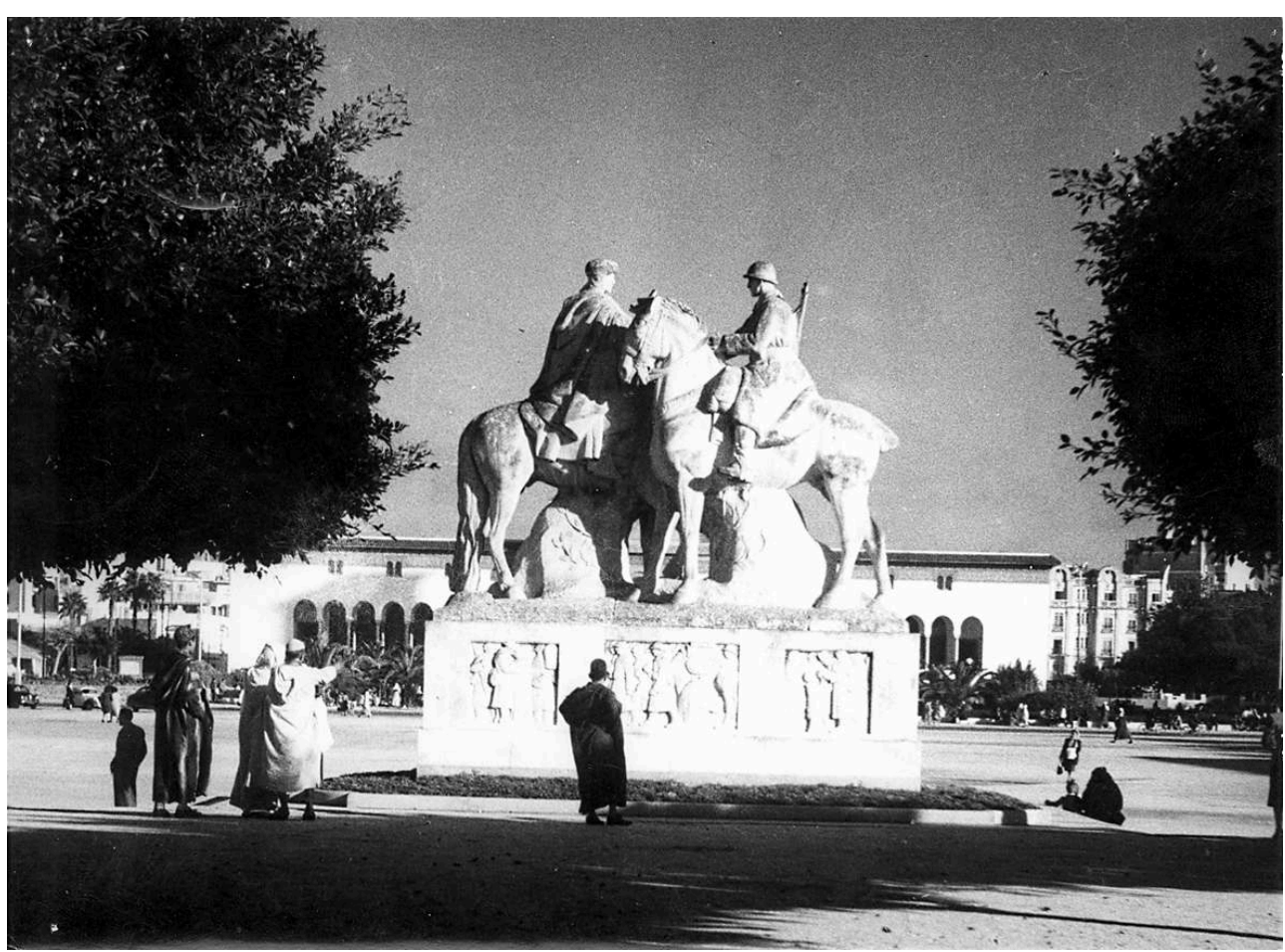

LE MONUMENT À LA VICTOIRE ET À LA PAIX, SUR SITE, AVEC LE PALAIS DE JUSTICE EN ARRIÈRE-PLAN, ANNÉEs 1950. PHOT. ROUgEt, BERNARD. COLLECTION PARTICULIÈre.

REPRO. ALEXANDRE, NICOLAS. (C) NICOLAS ALEXANDRE.

La dimension de l'œuvre de Landowski qui nous intéresse le plus ici est celle qu'il consacre à la commémoration de la Grande Guerre. Lui-même marqué par le conflit, combattant dans les tranchées de la Somme, récompensé par la Croix de Guerre, Paul Landowski réalise une trentaine de monuments en France métropolitain $\mathrm{e}^{14}$ en suivant la plupart du temps des thèmes iconographiques propres au genre : des représentations de " poilus », de «mères en deuil » ou plus rarement des allégories de la "Victoire »" Mais sa participation à l'œuvre commémorative est pour Landowski une charge qu'il ne compte pas subir. Ainsi, il écrit dans son journal le 4 décembre 1918: "Je suis formellement décidé si on me parle d'un de ces monuments à ne me charger de rien, à moins d'être absolument libre et d'avoir devant moi du temps ${ }^{16}$. Mais il concède un peu plus tard, le 12 janvier 1919 que « la vie est impossible». "Il faudra comme les autres m'atteler aux monuments de circonstances. Phidias dut bien, avant de faire le Parthénon, sculpter pendant longtemps des monuments commémoratifs des guerres médiques ${ }^{17} »$.

Malgré l'importance des commandes, il prend son temps. Son journal révèle la nature de son travail, ses hésitations, ses questionnements, ses recherches, même pour des monuments aux ambitions relativement modestes. Il montre aussi que l'artiste travaille 
à de très nombreuses œuvres en même temps. Ainsi, il est en perpétuelle ébullition. Lorsqu'il travaille au monument casablancais, il accepte le concours du monument d'Alger, travaille au monument de l'École normale supérieure, à celui de la Plaine de Chalmont et à beaucoup d'autres encore... Il le reconnaît lui-même et s'en enthousiasme: «Quelle vie magnifique que la vie d'artiste. Je me suis rarement senti mon cerveau en telle puissance $»^{18}$.

Figure 4

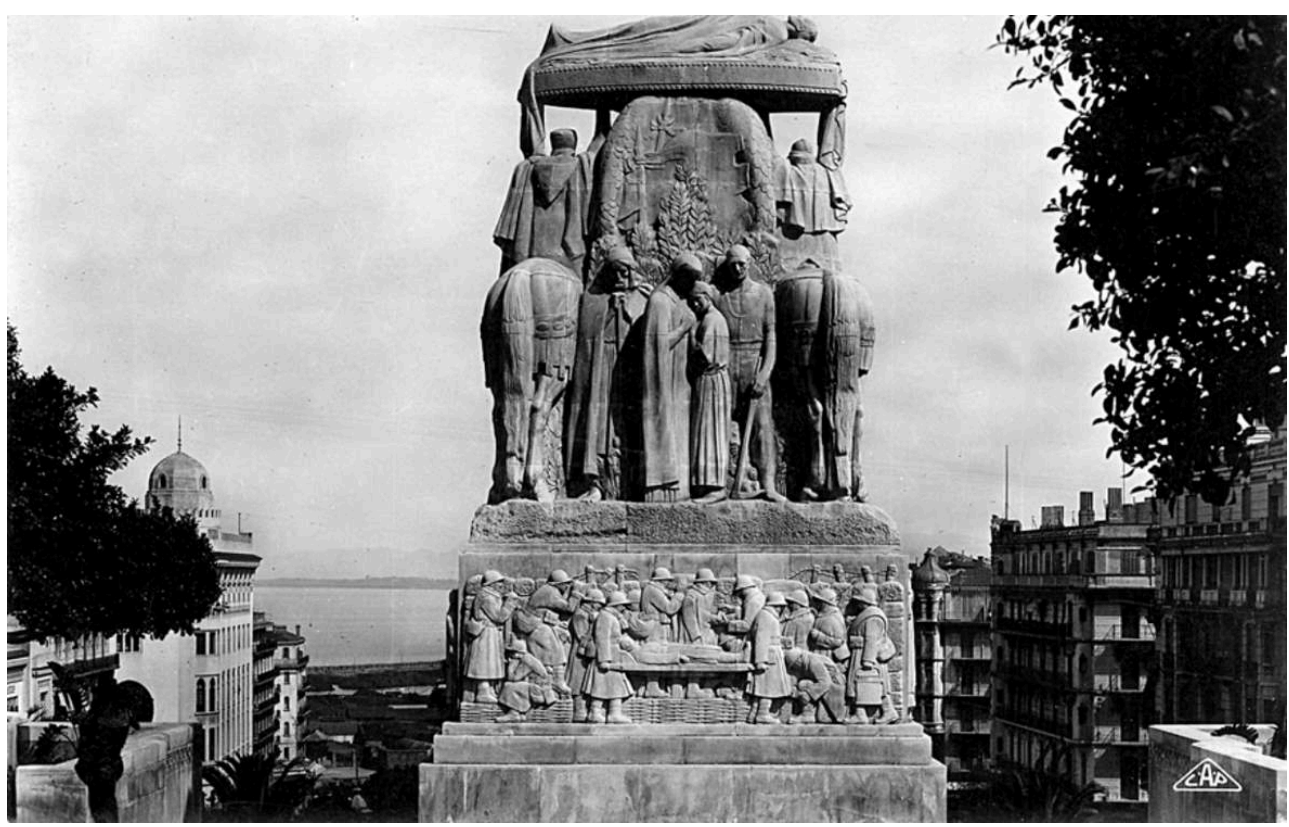

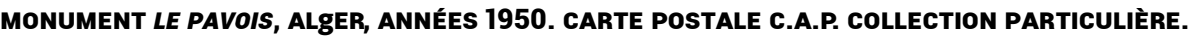

REPRO. ALEXANDRE, NICOLAS. (C) NICOLAS ALEXANDRE.

En fin de compte, cette "puissance » créatrice, c'est hors du territoire métropolitain que le sculpteur va la mettre au service de deux monuments commémoratifs d'envergure qui, avec l'ensemble de la Plaine de Chalmont (les Fantômes ${ }^{19}$, 1935), se détachent de l'ensemble des cénotaphes de Landowski : le Monument à la Victoire et à la Paix de Casablanca et celui d'Alger ou le Pavois. Ce dernier (fig. 4) pensé dès 1919, est achevé assez tardivement (1928). Commandé par la Ville d'Alger avec des ambitions comparables à celle de Casablanca, à savoir montrer l'étroite relation qui unissait les populations d'Europe et d'Afrique dans la victoire, le Pavois est d'une écriture beaucoup plus spectaculaire que le cénotaphe marocain. Cela relève des choix effectués: utilisation de l'allégorie antique de la Victoire ailée, assez rare chez Landowski, et traitement très médiéval de la scène. Le sculpteur était particulièrement intéressé par le Moyen Âge qui marque la scène par la présence de la silhouette du gisant mais aussi des chevaux caparaçonnés et décorés. Enfin, la modernité est représentée par les deux cavaliers contemporains (européen et africain). Ce monument vient donc démontrer le « souci de syncrétisme dans l'œuvre de Landowski : l'Antiquité, le Moyen Âge, le monde contemporain réunis pour viser à une image symbolique intemporelle dépassant la commémoration ponctuelle $»^{20}$. Ce monument montre surtout l'attachement de Landowski aux travaux de grande envergure. 
Le travail sur le monument casablancais se veut moins spectaculaire, avec une réflexion sur une scène plus familière dans laquelle il peut tout autant exprimer sa conception de la sculpture.

Landowski est ainsi persuadé de la haute mission de l'artiste devant la collectivité, et au-delà devant l'éternité. Mission d'autant plus noble selon lui, qu'elle doit témoigner des aspirations les plus hautes, et non plus des bassesses humaines d'où l'absence de tout dolorisme. De plus, Landowski considère que l'art, enjeu social, est destiné au plus grand nombre et aux petites gens en priorité. L'œuvre doit être accessible à tous ${ }^{21}$.

\section{Description du Monument de la Victoire et de la Paix}

L'ensemble a été réalisé entre 1921 et 1923 par le sculpteur en pierre de Lens ${ }^{22}$ pour les statues équestres et en marbre de l'oued Yquem ${ }^{23}$ pour le socle. Il existe une esquisse ${ }^{24}$, assez différente de la version finale, une maquette ${ }^{25}$ ainsi que des plâtres chamottés ${ }^{26}$ qui témoignent du travail préparatoire de l'artiste : c'est dans ce processus de genèse que s'inscrivent les plâtres que nous avons découverts à Casablanca en juin 2014 (fig. 5)

Figure 5

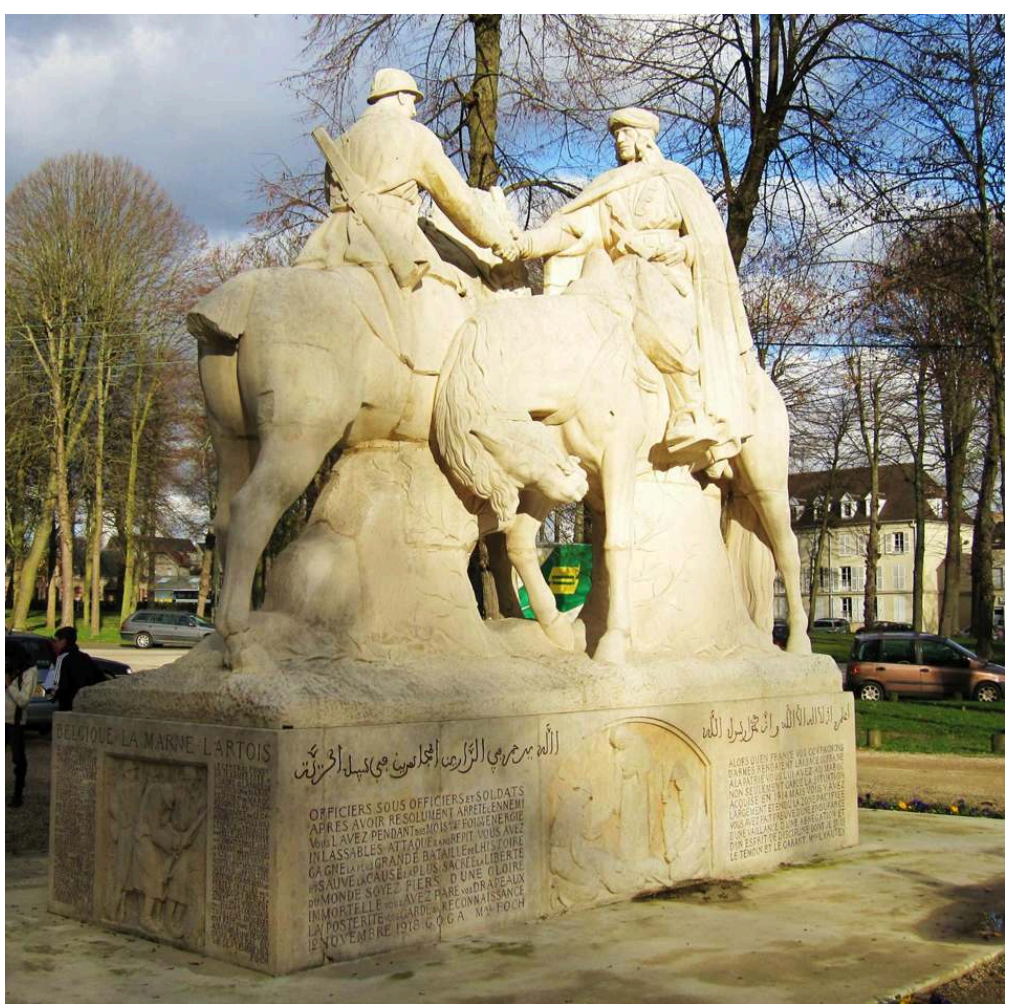

LE Monument à la Victoire et à la Paix, à Senlis. (Voir note 22). Photographie publiée sur le site http:// monumentsmorts.univ-lille3.fr/monument/7995/senlis-cimetiere/.

\section{Landowski}

a recours à un procédé qu'il utilise souvent : la ronde bosse qui symbolise et le basrelief qui raconte. Ici la ronde bosse exprime l'amitié entre les deux communautés incarnées par un cavalier français et un spahi marocain venant à la rencontre l'un 
de l'autre et se tendant la main. Les bas-reliefs du socle évoquent quant à eux, les scènes de guerre ${ }^{27}$.

\section{Les statues équestres}

En ronde bosse, la partie supérieure du monument représente deux cavaliers se faisant face et se serrant la main. D'un côté, un soldat français en uniforme de soldat avec un casque Adrian classique, modèle qui s'est imposé à partir de 1915, fusil en bandoulière, capote et ceinture à cartouchière, pantalon culotte, guêtres qui recouvrent des bandes molletières pour arriver jusqu'aux brodequins; de l'autre, un spahi en uniforme d'apparat, chéchia de feutre cachée par un empilement de chèches qui laisse apparaître les nouaders ${ }^{28}$, burnous sur une veste, cartouchière avec son brêlage, sarouel traditionnel de forme arabe, jambières de cuir (houseaux) permettant de protéger ensuite les jambes jusqu'aux brodequins. Le cheval du soldat français lève la tête tandis que celui du spahi la baisse, ce que certains ont interprété comme un signe de soumission ${ }^{29}$; mais la poignée de main est ferme, les bustes des hommes droits et leurs visages posés se regardent franchement : on peut supputer que le cheval baisse la tête pour permettre au spectateur de voir le geste central de la composition, à savoir la poignée de main. Notons que les supports des deux chevaux sont agrémentés de rameaux d'olivier qui symbolisent la paix retrouvée grâce à l'entraide des deux soldats. On peut aussi remarquer que la queue du cheval du soldat français est coupée tandis que celle du spahi ne l'est pas : ce détail, qui n'apparaît pas sur les plâtres sculptés du musée-jardin Paul Landowski mais est visible sur la photographie du monument réalisée lors de son inauguration, peut être imputé soit au hasard - la queue s'étant cassée lors de la réalisation de l'œuvre, soit à la volonté de traduire des usages différents selon les cultures, la caudectomie étant fréquente au début du XX $\mathrm{XX}^{\mathrm{e}}$ siècle en Europe.

Landowski a été inspiré par une scène vécue. Il raconte dans son journal :

Tout en nous promenant, l'idée me vint soudain de réaliser là une scène que j'ai vue, voici bien longtemps, un jour, dans la campagne romaine. Deux gardiens de troupeaux, à cheval, se rencontrant, vinrent à la rencontre l'un de l'autre et se donnèrent la main par dessus l'encolure de leur monture. Geste symbolique. Rapidement, je fais un petit croquis. Je le montre à Prost et lui explique mon idée ${ }^{30}$.

Quelques années plus tard, il commente ainsi cette réminiscence :

Il semble que la scène se soit fixée dans ma mémoire pour ressortir au moment voulu, comme d'un carnet de croquis. Mais pourquoi cette scène est-elle venue se placer sur ce sujet du monument de la Victoire? Un peu pour les mêmes raisons équestres que le monument d'Alger. Suite d'associations d'idées qui amènent à la trouvaille ou ressuscitent les souvenirs ${ }^{31}$.

Cette réflexion de l'artiste tempère en quelque sorte le message politique du monument: si Landowski relie ici le monument d'Alger et celui de Casablanca à un souvenir de la campagne romaine, on peut y voir une des marques, fréquentes à cette époque, d'exotisme teinté de poésie qui sublime la simple fonction commémorative du monument.

\section{Les six bas-reliefs}

Ces bas-reliefs sont l'objet principal de notre article car nous avons pu découvrir à Casablanca, en juin 2014, les six plâtres sculptés qui ont servi à leur réalisation pour le 
monument. Les plâtres sont exposés sur les murs d'une cage d'escalier, soutenus par des crochets, dans un lieu abandonné et menacé de destruction ${ }^{32}$ (fig. 6). Leurs dimensions sont identiques à celles du monument achevé : il s'agit donc des dernières esquisses de l'artiste avant la réalisation finale. Leur état de conservation est variable : quatre sont en bon état, un est en partie mutilé en son centre, un autre est tronqué sur sa partie droite.

Figure 6

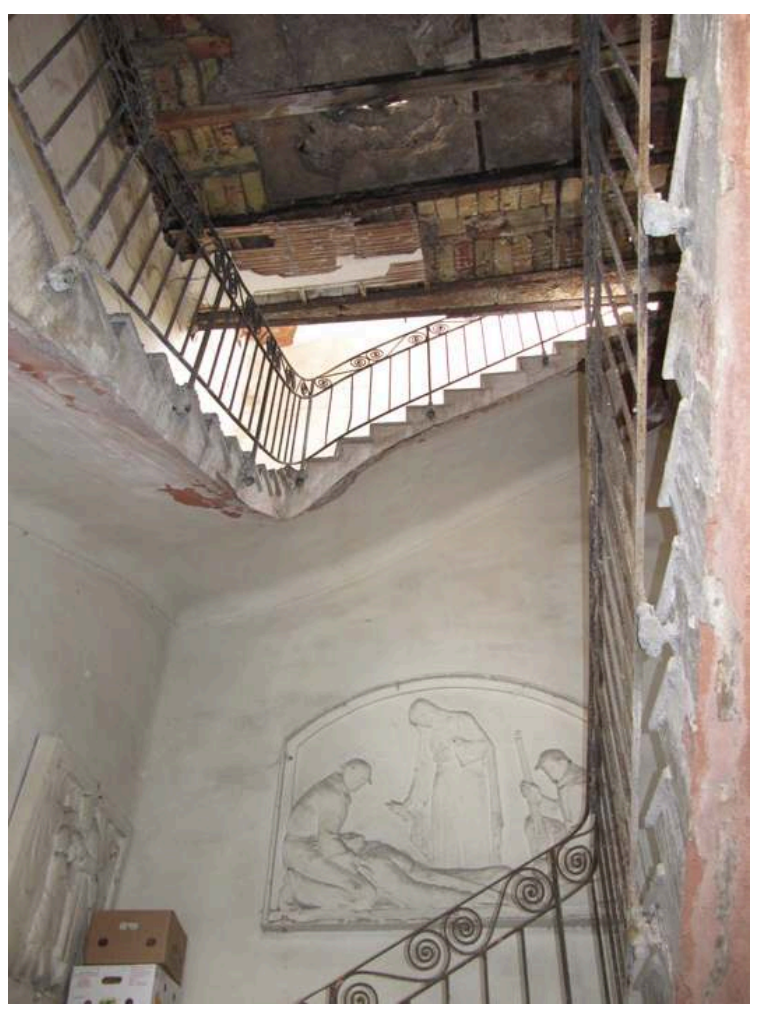

LA CAgE D'escalier où sont exposés les plâtres des bas-reliefs du Monument à la Victoire et à la Paix. PHot. Nicolas, Alexandre et Neiger, Emmanuel, juin 2014. (C) ALEXANDRE nicolas ET EMmanuel NEIgER.

Ceux-ci illustrent la coopération franco-marocaine en six scènes, citées par la revue France-Maroc ${ }^{33}$ :

- «L'Hommage aux morts» (fig. 7) : La scène la plus grande et qui est entourée de textes, en français et en arabe, représente une scène d'hommage composée de quatre personnages, trois soldats et une femme. Les hommes en uniforme de combattant, portant le classique casque Adrian et la capote Poiret, sont agenouillés; l'un à gauche soutient la tête du soldat mort, l'autre en face de lui à droite tient un fusil. Une femme, symbolisant à elle seule l'ensemble des veuves de guerre, se tient debout au centre de la scène, le visage incliné dans une pose pathétique et vient déposer une branche de laurier sur le corps du défunt, en hommage au héros mort pour la France. Il s'agit sans doute aussi d'une véritable allégorie de la Victoire rendant hommage à ses morts. On peut d'ailleurs souligner que sur la version définitive du bas-relief est rajouté un drapeau en arrière-plan qui n'apparaît pas sur le plâtre que nous avons découvert. 


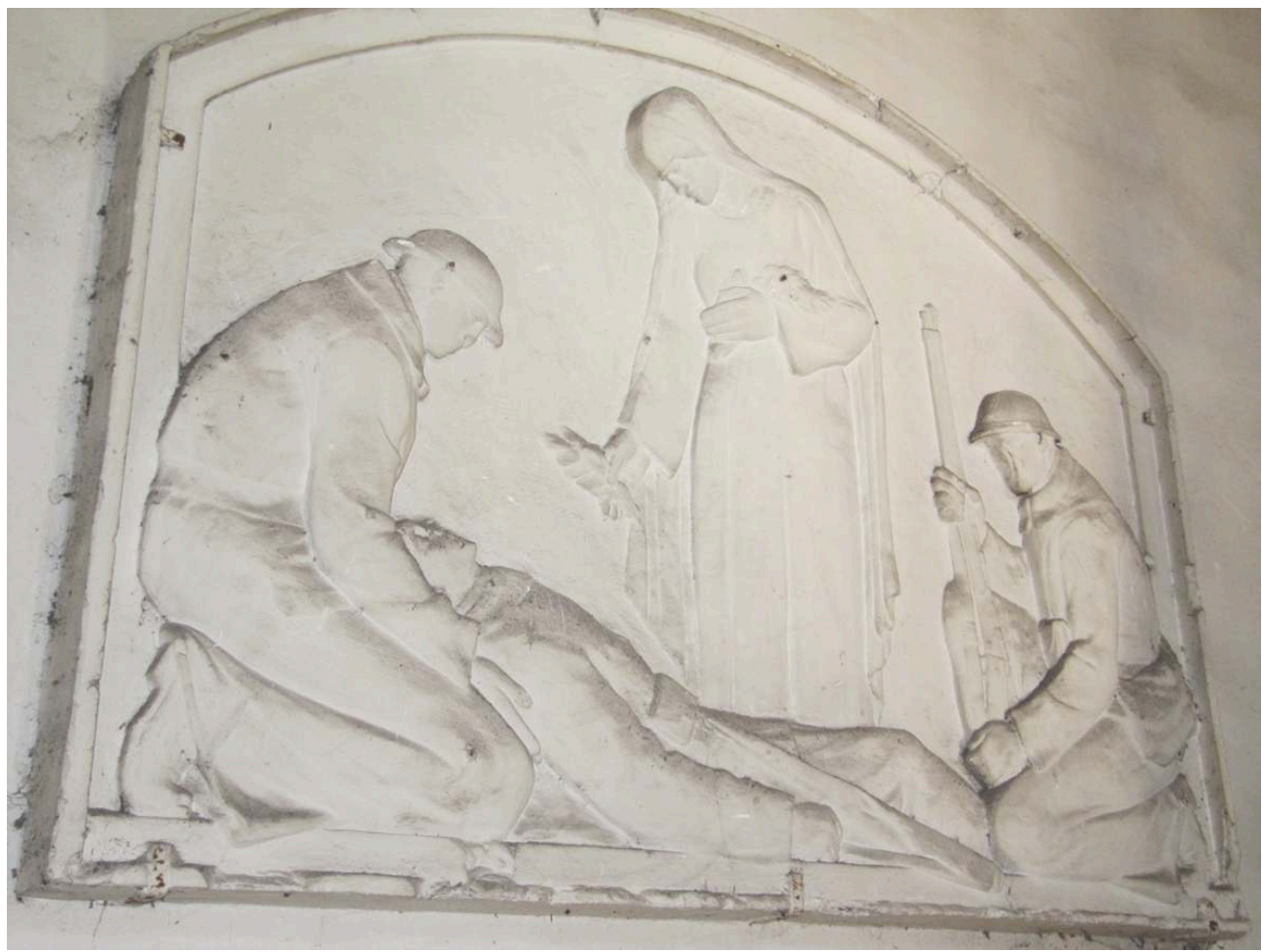

« L'Hommage aux morts ».

PHot. Nicolas, Alexandre et Neiger, Emmanuel, juin 2014. @ ALEXANDRE Nicolas et EMmANuel NEIgER.

Les autres scènes représentent des soldats français et marocains dans différentes situations. Tout d'abord, trois scènes occupent la longue face du socle opposée à la scène précédente et sont séparées par deux panneaux où est gravée une liste des régiments ayant combattu en France :

- « Le départ » (fig. 8), à gauche : les soldats en armes avec leur barda partent au front ; l'un d'eux dit adieu à une femme, sans doute son épouse, au centre de la scène. 


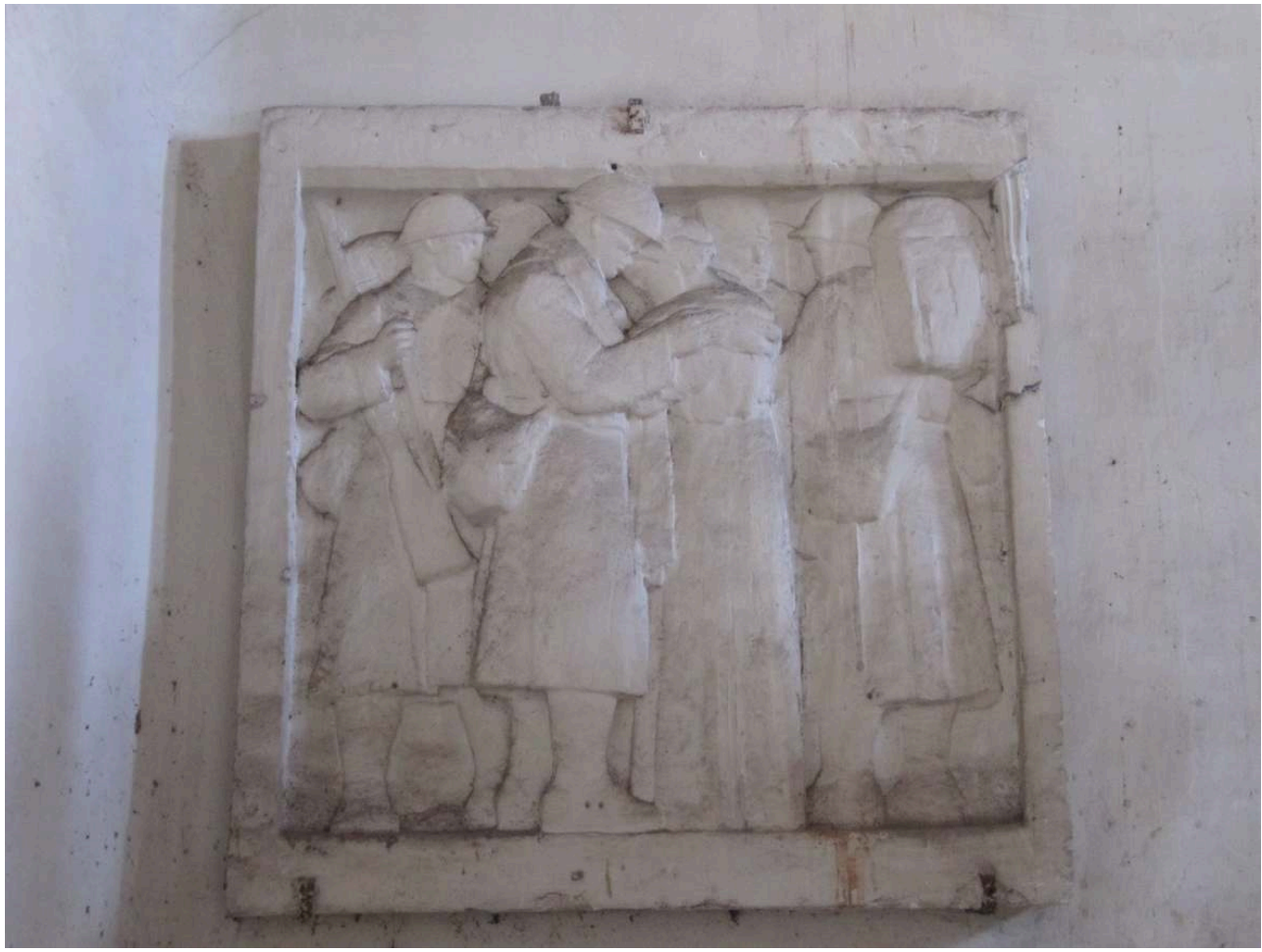

«Le départ ».

PHot. Nicolas, Alexandre et Neiger, Emmanuel, juin 2014. (C) ALEXANDRE nicolas ET EMmANuel NEIgER.

- « En colonne » (fig. 9), au centre : la montée des soldats, français et marocains, au front avec leur barda et des animaux portant du matériel, dont un dromadaire; les uniformes différents, tenues de spahis avec chèches et burnous ou de "poilus » avec casques et capotes servent à montrer l'épreuve partagée et attendue de la montée au front. 
Figure 9

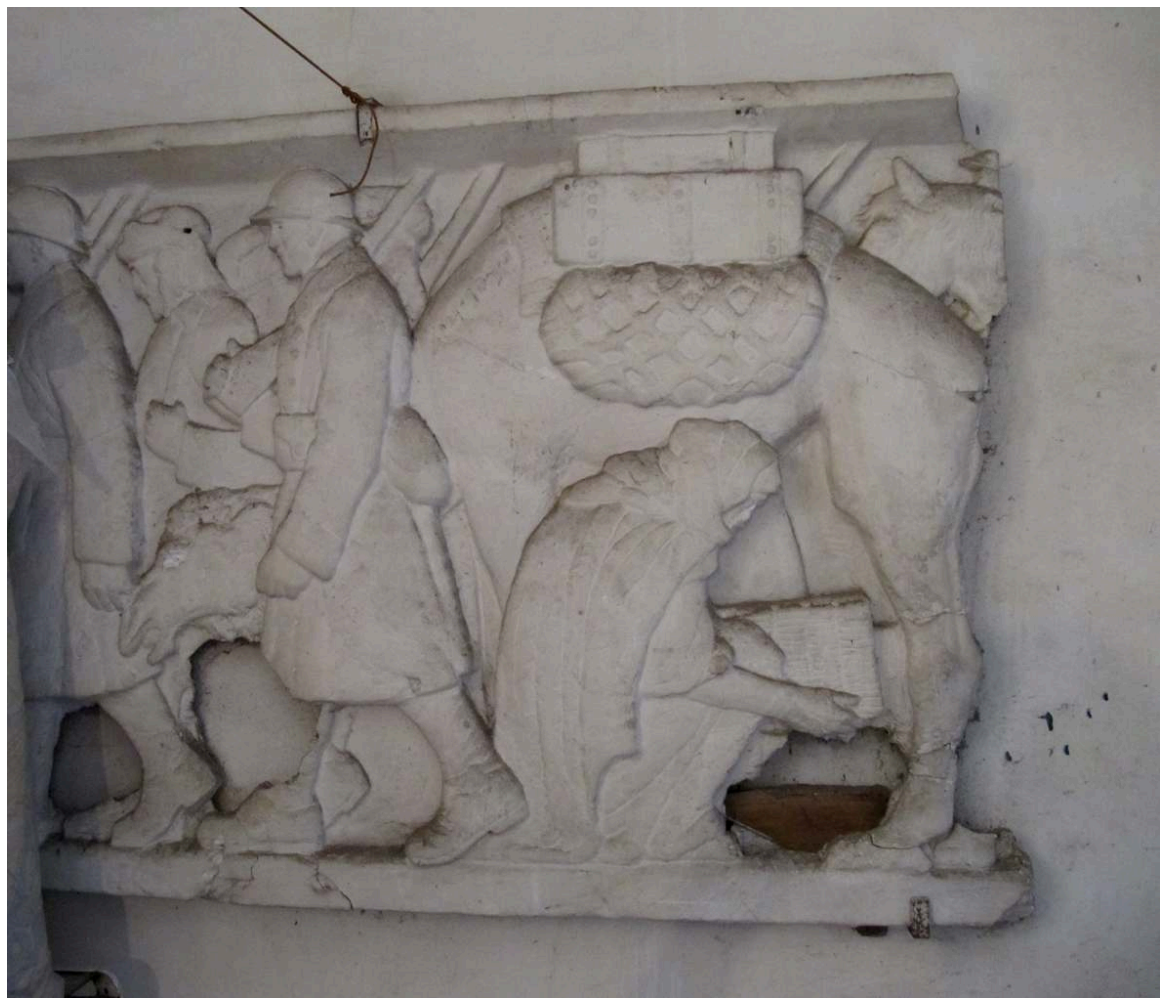

«En colonne».

PHot. Nicolas, Alexandre et Neiger, Emmanuel, juin 2014. (C) ALEXANDRE Nicolas ET NEIgER EMMANUEL.

« La Paix » (fig. 10) : une scène de retour de soldats accueillis par des femmes et des enfants en tenue traditionnelle ; une petite fille donne un bouquet à un soldat portant encore son paquetage.

Figure 10

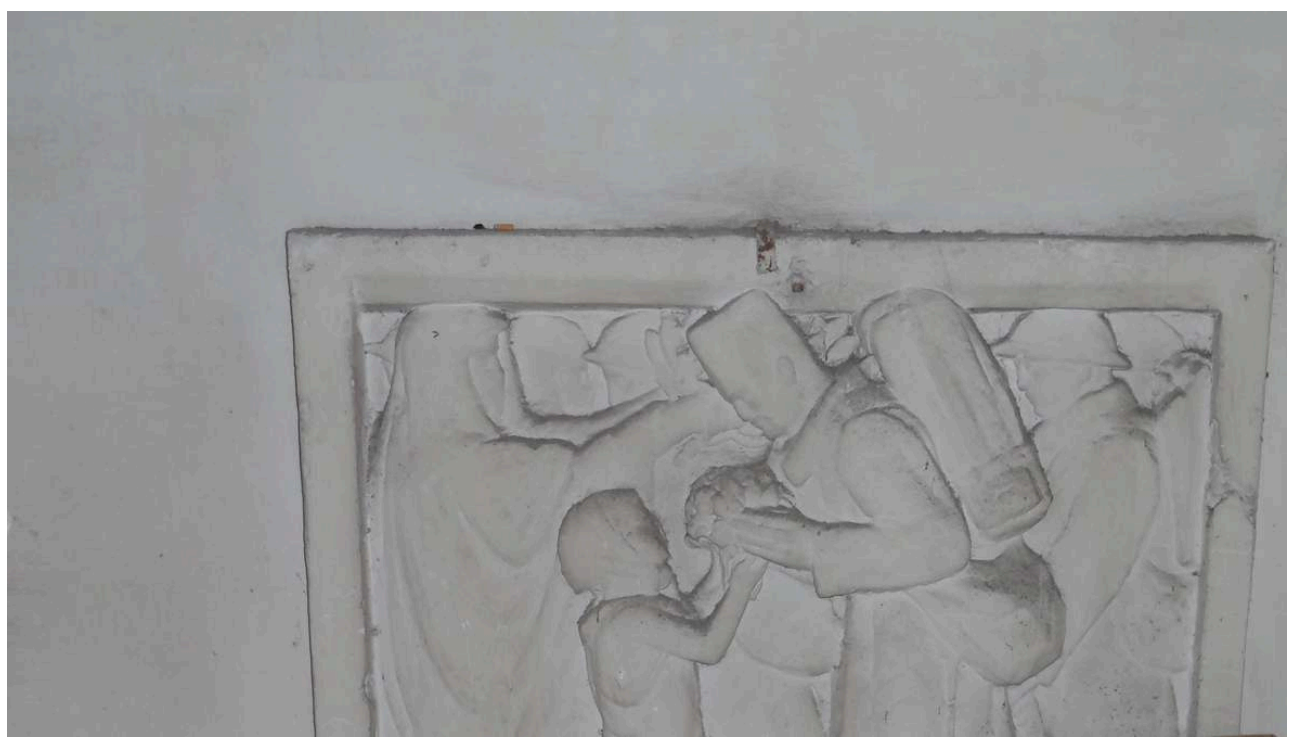

"La Paix ».

PHot. Nicolas, Alexandre et Neiger, Emmanuel, juin 2014. (C) ALEXANDRE Nicolas ET EMmANuel NEIgER. 
Enfin, aux deux extrémités du socle, dans sa largeur, se trouvent deux scènes :

- «La guerre des tranchées" (fig. 11): scène de chantier avec soldats français et marocains creusant une tranchée. Les torses nus et les bras noueux de deux personnages soulignent, dans une esthétique réaliste, la force et l'effort, tandis que la composition suggère le mouvement grâce aux lignes obliques marquées par les manches des outils. Ce bas-relief est flanqué de deux inscriptions qui relatent, pour celle de gauche portant la signature de Lyautey, une victoire au Maroc dans la région de Bou Denib et, pour celle de droite, une victoire des tirailleurs marocains en France.

Figure 11

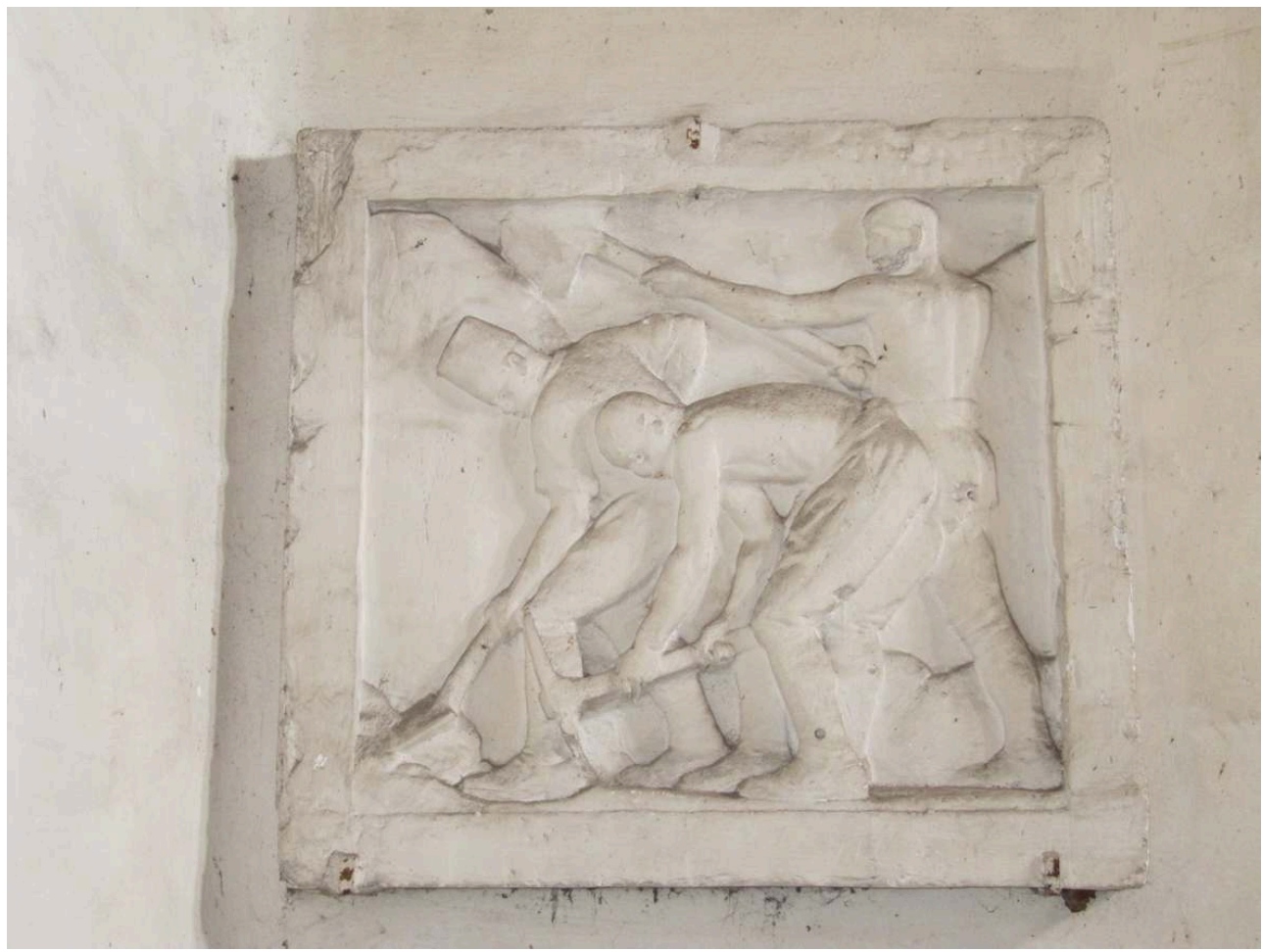

«La guerre des tranchées ».

PHot. Nicolas, Alexandre et Neiger, Emmanuel, juin 2014. (C) ALEXANDRE nicolas Et EMmanuel NEIgER.

- «La Victoire » (fig. 12) : une scène de combat avec des soldats en armes; les hommes armés de fusils avec baïonnettes sont dans la tranchée face au parapet; en train de recharger leurs fusils, ils se préparent sans aucun doute à l'assaut, l'épreuve tant redoutée par les soldats des tranchées. Cette scène est entourée dans le monument de deux bandeaux de texte qui narrent plusieurs batailles où se sont illustrées les troupes marocaines aux côtés des soldats français avec comme inscription supérieure: «La Belgique, La Marne, L'Artois ». 


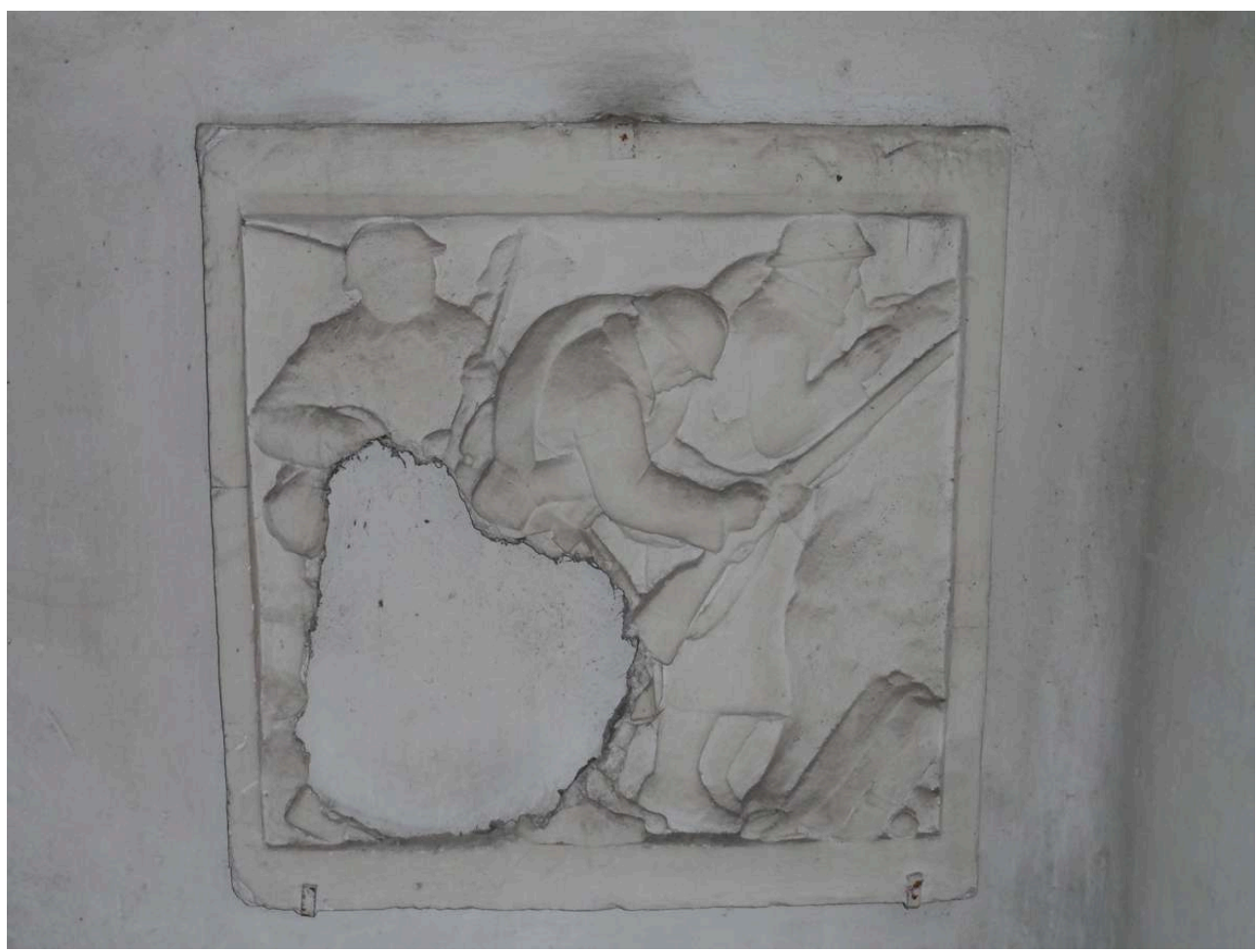

"La Victoire».

PHot. Nicolas, Alexandre et Neiger, Emmanuel, juin 2014. (C) ALEXANDRE Nicolas Et EMmanuel NEIgER.

\section{Le texte (gravé en français et en arabe)}

Voici la transcription du texte entourant la scène principale, « L'Hommage aux morts ", gravé en français :

- à gauche du bas-relief : "Officiers, sous-officiers et soldats, après avoir résolument arrêté l'ennemi, vous l'avez, pendant des mois, avec une foi et une énergie inlassables, attaqué sans répit. Vous avez gagné la plus grande bataille de l'histoire et sauvé la cause la plus sacrée : la liberté du monde. Soyez fiers. D'une gloire immortelle, vous avez paré vos drapeaux. La postérité vous garde sa reconnaissance, 12 novembre 1918, GQGA $^{34}$ Mal Foch $»^{35}$.

- à droite du bas-relief : "Alors qu'en France, vos compagnons d'armes rendaient l'Alsace-Lorraine à la patrie, vous lui avez, au Maroc, non seulement gardé la situation acquise en 1914 mais vous y avez largement étendu la zone pacifiée. Vous avez fait preuve d'une endurance, d'une vaillance, d'une abnégation et d'un esprit de discipline dont je suis le témoin et le garant. Mal Lyautey ${ }^{36}$ ".

Ces inscriptions, ainsi que celles qui encadrent la scène "La guerre des tranchées », appellent un commentaire : ce monument, placé à Casablanca, ne se contente pas de commémorer la guerre de 1914-1918. En effet, si les combats en France sont évoqués de même que le message du généralissime, la politique de "pacification » menée par Lyautey au Maroc y figure également. Cela prouve encore une fois l'enjeu politique de ce monument au cœur de la place administrative. 


\section{Le devenir du monument ou la question du patrimoine colonial}

Avec l'indépendance du Maroc, la question est posée du maintien des monuments marquants de la présence coloniale sur la place administrative : la statue équestre de Lyautey réalisée par François Cogné et le Monument à la Victoire et à la Paix de Landowski. Ainsi, suite aux accords de la Celle-Saint-Cloud (novembre 1955), la statue de Lyautey est déplacée à l'intérieur du jardin de l'Hôtel de la Subdivision militaire qui est, depuis 1959, le Consulat Général de France.

Le Monument à la Victoire et à la Paix est quant à lui démonté à partir de $1961^{37}$ et remis officiellement à la ville de Senlis le 11 novembre 1965, comme l'indique une plaque sur place dont voici le texte :

Monument dit de la Victoire ou de la Fraternité Franco-Marocaine édifié à Casablanca à partir de 1921, inauguré le 20 juillet 1924 par le Maréchal Lyautey, démonté en 1961 par les soins du consulat général de France, transporté à Senlis par la Marine et par l'Armée, réédifié sur cette place par les soins de l'armée de la ville de Senlis et de l'association Le Burnous, remis le 11 novembre 1965 au maire de Senlis M. Yves Carlier par le président du Burnous, le Général Yves Simon.

Le démontage et la réédification font l'objet d'une négociation et finalement d'une entreprise commune ${ }^{38}$ menée par le Consulat général de France à Casablanca, l'Armée, la Ville de Senlis et l'Association Le Burnous ${ }^{39}$. Le choix de la ville de Senlis et de l'emplacement exact désigné pour accueillir le monument, la place du $3^{\mathrm{e}}$ Houzards, sont liés notamment à la localisation de régiments de spahis dans cette ville ${ }^{40}$. Le monument rebaptisé Monument de la fraternité franco-marocaine est donc implanté devant leur ancienne caserne devenue en 1964 celle d'une unité de blindés légers.

C'est également en mai 1961 que les cendres du maréchal Lyautey sont rapatriées en France pour qu'il soit inhumé aux Invalides ${ }^{41}$. Le contexte est celui de la fin de la guerre d'Algérie, des mouvements d'indépendance dans toutes les anciennes colonies françaises, et d'une politique du général De Gaulle qui vise à reconnaître progressivement les revendications des peuples à la liberté comme il l'affirme dans le discours qu'il prononce à l'occasion de la cérémonie d'accueil des cendres du maréchal Lyautey aux Invalides :

Politique, ce n'est pas du tout à l'abaissement d'un empire et à la domination d'un pays qu'il tendit son action de résident général de France au Maroc, mais au contraire à la consolidation d'un État souverain, au développement d'une élite et d'un peuple pour les aider à devenir capables de porter un jour les responsabilités de l'indépendance et de la civilisation ${ }^{42}$.

De leur côté, les Marocains appréciaient modestement ce monument qui symbolisait pour eux, comme tous les monuments de l'époque du protectorat, une présence coloniale, d'autant que le principe même de monuments de commémoration semble étranger à la culture locale. Il paraît donc naturel qu'il ait fait l'objet d'une forme de rejet, d'autant qu'il était placé au centre d'une place éminemment française.

Le devenir du Monument à la Victoire et à la Paix amène donc à s'interroger sur la question des indépendances et de la manière dont les pays enfin libres vivent leur passé colonial.

Ainsi, à Alger, Le Pavois, œuvre de Landowski également, a connu un destin différent du cénotaphe de Casablanca. Il n'est plus visible aujourd'hui : il a été recouvert d'un 
sarcophage de béton à la demande des autorités algériennes à la veille des Jeux africains de 1978 par l'artiste M'hamed Issiakhem qui a semble-t-il tout fait pour protéger l'œuvre de Landowski ${ }^{43}$.

Figure 13

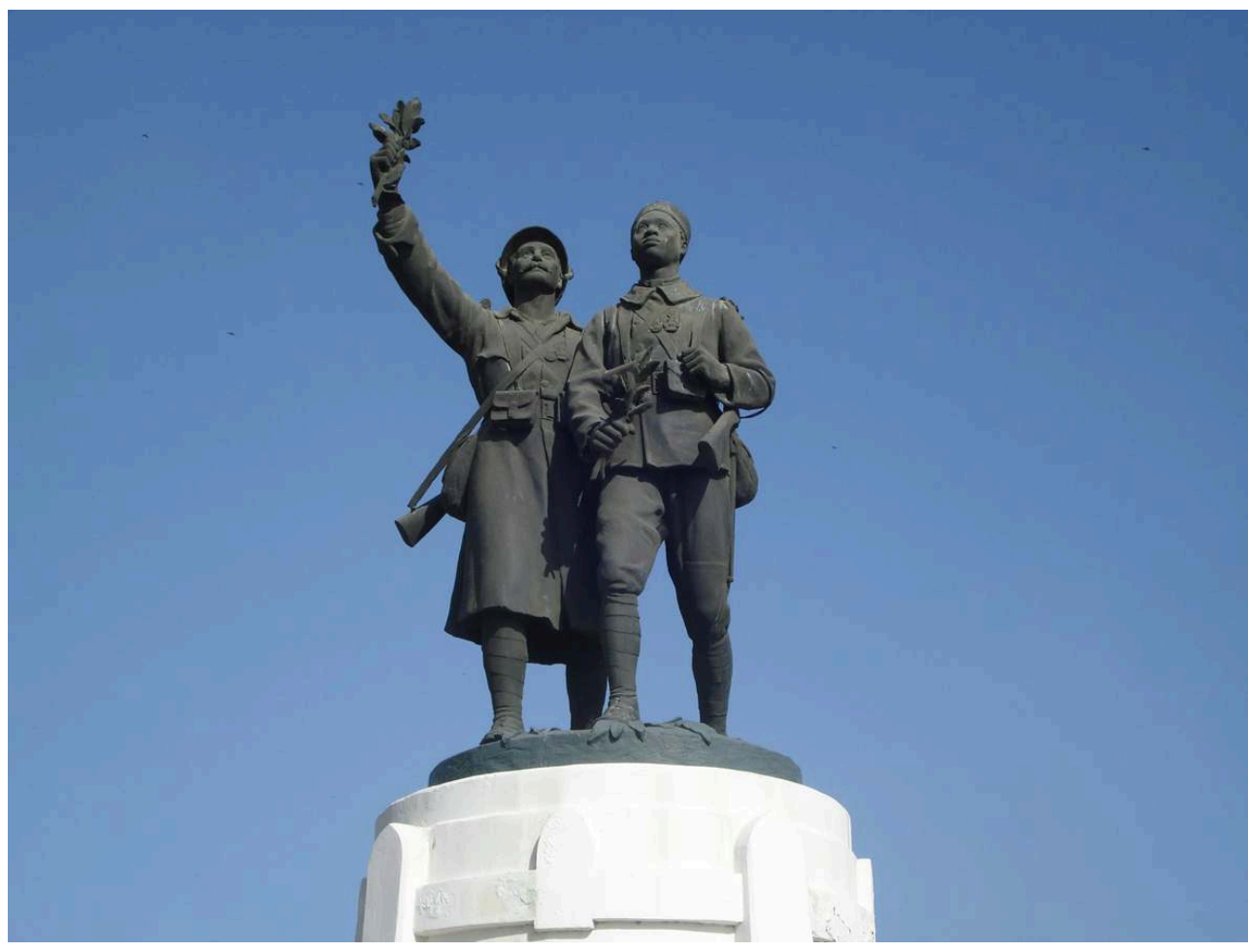

Monument À la gloire des troupes noires et aux créateurs disparus de l'Afrique occidentale française, Dakar.

Phot. Alcaix, Gil. (C) Gil Alcaix, publiée sur le site http://dictionnaireduchemindesdames.blogspot.com/ 2008/11/t-comme-tirailleurs-sngalais.html.

D'autre part, à Dakar, un monument aux morts, situé à l'époque sur le rond-point de l'Étoile, dans le nouveau quartier européen des années 1920, proche du quartier militaire et de l'hôpital, a un destin plus complexe (fig. 13). Exécuté en bronze en 1923 par le sculpteur français Paul Ducuing ${ }^{44}$, ce monument À la gloire des troupes noires et aux créateurs disparus de l'Afrique occidentale française célèbre deux soldats, Demba, le tirailleur africain, et Dupont, le poilu français ${ }^{45}$. Ils reposent sur un piédestal où figurent des bas-reliefs de cinq personnalités importantes de l'AOF : Faidherbe ${ }^{46}$, Griffon du Bellay ${ }^{47}$, William Merlaud-Ponty, François Joseph Clozel et Josst Van Vollenhoven ${ }^{48}$. Le sculpteur a voulu, comme Landowski sur le Monument à la Victoire et à la Paix mettre en évidence le lien de fraternité qui a uni les combattants africains et français au cours de la Grande Guerre. Le fait que les deux soldats regardent dans la même direction, indiquée également par le rameau d'olivier levé par le soldat français, suggère la communauté de destin des deux frères d'armes. Mais certains verront également dans la main posée sur l'épaule du Tirailleur un geste paternaliste. Pourtant, il faudra attendre 1983 pour que Demba et Dupont soient emportés avec la statue de Faidherbe. Comme dans de nombreux pays ayant acquis leur indépendance, mais avec beaucoup de retard puisque il s'est totalement émancipé en 1960, le Sénégal a voulu enlever certains symboles de la présence coloniale ${ }^{49}$. Figures d'un passé révolu, Demba et Dupont furent déboulonnés dans la nuit du 13 au 14 août 1983 déplacés et 
abandonnés dans le cimetière catholique et militaire de Bel Air à Dakar. Ce fut la trace la plus visible d'un mouvement conduisant à rebaptiser rues et établissements d'enseignements. Ainsi, nous pouvons lire dans une revue sénégalaise de 1983 :

Leur symbolisme originel était mort, et leur présence blessait l'orgueil national de nombreux citoyens. Les autorités sénégalaises ont décidé de les transférer dans le futur musée d'histoire que l'armée se propose de créer. Ils y seront les témoins d'une période importante de l'héritage national, celle de l'occupation coloniale. L'exemple sénégalais pourrait être suivi par d'autres pays de l'Afrique libre, indépendante et maîtresse de son devenir ${ }^{50}$.

L'état d'esprit est donc bien moins radical que dans les cas casablancais (déménagement) ou algérois (recouvrement). Le monument reste à Dakar et on envisage même de le mettre dans un musée. C'est en 2004 que la sculpture de Ducuing va connaitre sa seconde vie par la décision du président sénégalais Abdoulaye Wade ${ }^{51}$. Lors de la célébration de la Journée du Tirailleur, inaugurée le 23 août 2004, il ${ }^{52}$ fait réinstaller la statue au centre de Dakar, place de la Gare Dakar-Niger, rebaptisée à cette occasion Place du Tirailleur. Un monument commémoratif situé à proximité immédiate porte désormais gravé : "À nos morts, honneur et reconnaissance éternelle de la Nation ". Soucieux de rendre aux Tirailleurs leur juste place dans l'histoire nationale sénégalaise, il leur a dédié, cette même année, la journée du 23 août, en souvenir de la libération de Toulon intervenue quelques semaines après le débarquement de Provence en 1944. Le nouvel emplacement choisi, situé face à la gare Dakar-Niger et face à l'embarcadère de Gorée est symbolique dans la mémoire des Anciens Combattants : la place était en effet le point de passage des soldats qui partaient rejoindre le front en Europe. Dans un entretien au journal Le Monde, en 2008, le président Wade justifie ainsi sa décision :

Elle [la double statue] avait été reléguée dans un cimetière. Quand je suis arrivé au pouvoir, j'ai demandé qu'on aille la chercher car, pour moi, elle représente un pan de notre histoire. Il faut assumer l'histoire, quelle qu'elle soit ${ }^{53}$.

Les destins si différents de ces trois monuments aux morts de la guerre de 14-18 traduisent donc des divergences dans la manière dont les pays anciennement colonisés abordent leur propre histoire et leur rapport à la France. Les monuments aux morts réalisés par des artistes français et installés par les autorités coloniales dans un esprit de commémoration mais aussi d'affirmation de la présence étrangère constituent bien un patrimoine vivant, qui interroge encore de nos jours le passé, l'histoire et le présent. Le déplacement de celui de Casablanca, le recouvrement de celui d'Alger ou la nouvelle mise en scène de celui de Dakar prouvent à quel point les monuments aux morts interpellent et constituent des éléments signifiants qu'on ne détruit pas mais auxquels on peut faire signifier, sans nécessairement les transformer, des messages différents, symptômes aussi bien de l'époque de leur édification que de celle où ils subissent une modification.

\section{Conclusion}

Le Monument à la Victoire et à la Paix de Paul Landowski constitue donc un témoignage éminent sur le rôle des troupes marocaines dans la Première Guerre mondiale. Mais il est aussi une réalisation artistique qui pose la question de ce qu'est un monument officiel : entre les désirs et inspirations de l'artiste et la mise en scène qui est faite de son œuvre de commande, un fossé se creuse. 
En outre, la mémoire du passé qu'incarne étymologiquement un monument devient un message politique pour le présent et pour l'avenir : le discours du résident général lors de l'inauguration montre clairement que le cénotaphe constitue un manifeste des vues de Lyautey.

C'est dire à quel point le Monument à la Victoire et à la Paix représente un patrimoine national pour le Maroc et pour la France, aussi bien en tant qu'œuvre sculptée par l'artiste Paul Landowski qu'au titre de mémoire de l'histoire commune des deux pays. Son inscription à l'inventaire des Monuments Historiques semble donc nécessaire.

Par ailleurs, nous attirons l'attention de nos lecteurs sur les plâtres que nous avons découverts. Le fait qu'ils aient été conservés à Casablanca, dans un lieu de nos jours certes délaissé mais dans une mise en scène qui relève de l'exposition, démontre qu'ils font partie intégrante de l'histoire de la France et du Maroc: il serait dommageable qu'ils disparaissent, accaparés par une personne ou engloutis dans les destructions qui menacent le quartier ${ }^{54}$ en question.

La solution cependant n'est pas évidente : la question de l'identité du propriétaire reste pour l'instant insoluble. Mais le fait que la statue de Lyautey réalisée par Cogné ainsi qu'une plaque commémorant le débarquement des troupes françaises à Casablanca en 1907 se trouvent actuellement au Consulat de France, laisse entrouverte, nous l'espérons, la possibilité que ces plâtres y soient également conservés.

\section{NOTES}

1. - Voir TOURON, Christophe, RIERA, Jean-Pierre. Ana, frères d'armes marocains dans les deux guerres mondiales. Casablanca : Éd. SensoUnico, 2014, p. 18 sqq.

2. - Voir l'analyse de ce plan dans : COHEN, Jean-Louis, ELEB, Monique. Casablanca, Mythes et figures d'une aventure urbaine. Casablanca : Éd. Hazan, 1998, Rééd. 2004, p. 49-85.

3. - Ibid., p. 92-102.

4. - Voir pour ce monument l'analyse proposée par PIERRE, Jean-Luc, voir le site: http:// www.cndp.fr/crdp-reims/ressources/brochures/blphg/bul26/jlpierre.htm.

5. - Un recensement de 1921 chiffre la communauté française de Casablanca à 21579 personnes et celle de Rabat à 7383 personnes. Selon Augustin Bernard, "Le recensement ne semble pas toujours avoir été fait avec beaucoup de soin et le chiffre d'Européens qu'il accuse dans certaines villes notamment Casablanca est dit-on inférieur à la réalité ». Voir BERNARD, Augustin. "Le recensement de 1921 dans l'Afrique du Nord ». Annales de Géographie, tome 31, n¹69, p. 52-58, 1922.

6. - Image publiée sur le site: http://www.bmsenlis.com/sitebmsenlis/galerie/picture.php?/ 9613/category/107.

7. - « Inauguration du monument de la victoire ». France-Maroc, juin-juillet 1924, nº 61, p. 103-105. Voir le site : http://gallica.bnf.fr/ark:/12148/bpt6k61066931.image.langFR.r=france\%20maroc.

8. - LYAUTEY, Hubert. Paroles d'action: Madagascar, Sud-Oranais, Oran, Maroc (1900-1926). Paris : Armand Colin, 1927, p. 409-413.

9. - Voir l'analyse suivante : RIVET, Daniel. Lyautey et l'institution du Protectorat au Maroc, 1912-1925. Paris : Éd. L'Harmattan, 1996, tome I, p. 175-181. 
10. - Au Maroc, le Makhzen (étymon de notre mot français magasin) désigne tout le pouvoir central organisé autour du roi et du palais.

11. - Le monument est censé commémorer «l'indissoluble collaboration, dans la paix, de la France protectrice et d'un Maroc qui réalise progressivement ses grandes destinées ", affirme un journaliste dans «Inauguration, à Casablanca, par le maréchal Lyautey, du monument à la victoire et à la paix ». L'illustration, 2 août 1924, $\mathrm{n}^{\circ} 4248$.

12. - Les photographies de l'époque publiées dans différents journaux le démontrent nettement. Voir « Inauguration du monument de la victoire ». France-Maroc, op. cit. p. 105.

13. - «Solennité de Casablanca ». L'Afrique du Nord illustrée, 2 août $1924, n^{\circ} 170$, p. 1 et p. 3. Voir le site :

http://gallica.bnf.fr/ark:/12148/

bpt6k5562419z.image.langDE.r=L\%27Afrique\%20du\%20Nord\%20illustr\%C3\%A9e. Notons que cette photographie semble relever de la pose, après le discours et donc, d'une mise en scène qui permet d'affirmer, en couverture de la revue, l'association de la population marocaine à l'événement, dans un lieu, la place Mohamed V, que les Marocains fréquentent très peu à l'époque du Protectorat, une ségrégation y étant établie de fait, sinon de droit.

14. - LEFRANÇOIS, Michèle. "Paul Landowski et la commande publique : entre contrainte et opportunité ». Dans Paul Landowski et la commande publique, sous la dir. de Caillet, E. et SINGER, J. Paris : L'Harmattan, 2014, p. 53.

15. - LEFRANÇOIS, Michèle. Paul Landowski : l'œuvre sculpté. Paris : Éd. Creaphised, 2009, p. 63.

16. - Extrait du journal de Paul Landowski (4 décembre 1918, cahier n06), voir le site : http:// journal.paul-landowski.com (consulté le 29 septembre 2014).

17. - Extrait du journal de Paul Landowski (12 janvier 1919, cahier $\left.n^{\circ} 06\right)$, voir le site : http:// journal.paul-landowski.com (consulté le 29 septembre 2014).

18. - Extrait du journal de Paul Landowski (20 août 1922 , cahier $\left.n^{\circ} 14\right)$, voir le site : http:// journal.paul-landowski.com (consulté le 29 septembre 2014).

19. - Très différent des monuments algérois et casablancais, Les Fantômes exprime clairement les souffrances du champ de bataille que Landowski a lui-même vécues dans la Somme en 1916. «Une sensation d'infinie tristesse se dégage de ces morts de granit, qui paupières closes se relèvent lentement des champs mortifères. La dramaturgie existe mais elle est sobre " (dans LEFRANÇOIS, Michèle. Paul Landowski : l'œuvre sculpté, op. cit., p. 64)

20. - Ibid., p. 63.

21. - LEFRANÇOIS, Michèle, op. cit., p. 56.

22. Extrait du journal de Paul Landowski (4 août 1922, cahier $n^{\circ} 14$ ), voir le site: http:// journal.paul-landowski.com (consulté le 2 octobre 2014) : "J'ai donné ordre aux carrières de Lens de faire l'expédition des pierres à Casablanca. Voilà le monument qui entre dans sa période d'exécution définitive». Photographie (fig. 5) publiée sur le site http://monumentsmorts.univ-lille3.fr/ monument/7995/senlis-cimetiere/.

23. - Extrait du journal de Paul Landowski (20 octobre 1923, cahier $\left.n^{\circ} 17\right)$, voir le site : http:// journal.paul-landowski.com (consulté le 2 octobre 2014) : "nous sommes allés voir aux environs immédiats de Rabat, des carrières de marbre dont le propriétaire offre les blocs nécessaires pour le socle du monument. C'est à l'embouchure de l'oued Yquem ».

24. - Voir le site : http://www.paul-landowski.com/casablanca.

25. - La maquette est visible sur le site: http://www.culture.fr/collections/resultats?

keywords=landowski+paul+casablanca\&sel_search_mode=tous_les_termes\&sel_filter_ortho=sel_filter_ortho\&sel_filter_syn=sel_filter_s 26. - Voir l'inventaire du musée-jardin Paul Landowski de Boulogne-Billancourt sur le site: http://www.boulognebillancourt.com/previous/fonds_musees/notice402.html.

27. - LEFRANÇOIS, Michèle. «Paul Landowski et la commande publique : entre contrainte et opportunité ", art. cit., p. 53.

28. - Longues mèches de cheveux ébouriffés qui retombaient longuement sur les côtés et qui étaient un symbole de courage chez les berbères ; on les trouve chez les spahis mais aussi chez les 
tirailleurs marocains. Voir: TOURON, Christophe, RIERA, Jean-Pierre, op. cit., p. 28. Landowski semble s'être intéressé à cette coiffe lors de son voyage au Maroc en 1921, puisqu'il sculpte sur plâtre un Guerrier de la Chaouia qui porte ces nouaders, voir le site: http:// www.boulognebillancourt.com/previous/fonds_musees/notice386.html.

29. - COHEN, Jean-Louis, ELEB, Monique, op. cit., p. 98-100.

30. - Extrait du Journal de Paul Landowski (19 mai 1921, cahier $\left.n^{\circ} 10\right)$, voir le site: http:// journal.paul-landowski.com (consulté le 2 octobre 2014).

31. - Extrait du journal de Paul Landowski (25 avril 1930, cahier $\left.n^{\circ} 27\right)$, voir le site : http:// journal.paul-landowski.com (consulté le 29 septembre 2014).

32. - La piètre qualité des photographies s'explique par le fait que nous avons dû être discrets lors de la découverte. Les lecteurs voudront bien nous en excuser.

33. - Les titres donnés aux scènes sont tirés d'un article publié sans nom d'auteur: "Le monument de la victoire ", France-Maroc, décembre 1921, n 61, p. 241. Voir le site: http:// gallica.bnf.fr/ark:/12148/bpt6k6213093j/f19.image.

34. - Grand Quartier Général des Armées Alliées.

35. - Ce texte est repris de l'ordre du jour de la Victoire adressé aux armées alliées par leur général en chef, le maréchal Foch, au lendemain de la signature de l'armistice de Compiègne.

36. - Ce texte est repris de l'ordre du jour de la Victoire adressé par le résident général aux troupes stationnées au Maroc le 11 novembre 1918.

37. - Si nous avons des informations sur les conditions du déplacement du monument, rien pour l'instant ne vient éclairer le contexte de la prise de décision et le décalage entre l'indépendance, 1956, et le déclenchement du processus qui provoque le départ du monument vers Senlis, en 1961. Le décès du roi Mohamed $\mathrm{V}$ cette même année et le contexte lié au changement de monarque ont-ils influencé la décision? La question reste ouverte.

38. - Le transport depuis le Maroc est pris en charge par l'Armée de Terre ; la réédification menée à partir de juin 1965 est réalisée par les services du Génie de Laon ; l'Armée assure donc l'apport du matériel de levage et la main d'œuvre; l'Association Le Burnous prend en charge l'assurance du matériel et le carburant, la Ville de Senlis, la fourniture des matériaux et la direction des opérations. Voir le site: http://www.bmsenlis.com/sitebmsenlis/jp/index.php/dossiersdocumentaires/195-le-monument-de-l-amitie-franco-marocaine.

39. - Association créée en 1895 qui a pour objectif « de maintenir vivants entre eux les liens de camaraderie et d'entraide tissés sous l'uniforme » et « d'honorer les faits d'armes des Spahis et de leurs régiments et de perpétuer le souvenir de leur histoire ». Voir le site: http:// le.burnous.pagesperso-orange.fr/burnous/burnous.html.

40. - Le $4^{\mathrm{e}}$ Régiment de Spahis marocains stationné entre 1928 et 1939 et le $7^{\mathrm{e}}$ Régiment de Spahis algériens de 1948 à 1962.

41. - DONNADIEU, James. "Les cendres du maréchal Lyautey reposeront aux Invalides». L'impartial, 17 avril 1961, $\mathrm{n}^{\circ}$ 25568, p. 1-2. Voir le site: http://www.limpartialarchives.ch/ Repository/IMP/1961/04/17/113-IMP-1961-04-17-0001.pdf.

42. - Discours du Président de la République le 10 mai 1961, voir le site : http://www.charles-degaulle.org/pages/l-homme/accueil/discours/le-president-de-la-cinquieme-

republique-1958-1969/discours-du-10-mai-1961-a-l-occasion-de-la-ceremonie-d-accueil-descendres-du-marechal-lyautey-aux-invalides.php.

43. - Voir MEDDI, Adlène. "Patrimoine : le Pavois d'Alger se dévoile». El Watan 2, article du 25 octobre 2012 en ligne, voir le site: http://elwatanlafabrique.wordpress.com/2012/10/25/ patrimoine-le-pavois-dalger-se-devoile.

44. - Article du 29 mars 2010 sur le site internet de RFI, voir le site : http://www.rfi.fr/tirailleurs/ 20100329-statue-demba-dupont/ (consulté le 23 octobre 2014). Photographie (fig. 13) publiée sur le site http://dictionnaireduchemindesdames.blogspot.com/2008/11/t-comme-tirailleurssngalais.html. 
45. - « Demba et Dupont » semble être le nom rapidement donné par la population dakaroise au monument, relevant avec son humour habituel des noms de famille très répandus au Sénégal et en France.

46. - Louis Faidherbe (1818-1889), militaire, administrateur du Sénégal de 1854 à 1861 puis de 1863 à 1865 puis homme politique après la guerre de 1870-1871.

47. - Chirurgien de la Marine, « en 1861-1863, il sert sur l'hôpital flottant la Caravane, stationné au large du Gabon. (...) Il est le premier à avoir décrit la maladie du sommeil. » Voir le site du comité des travaux historiques et scientifiques : http://cths.fr/an/prosopo.php?id=112321\#.

48. - Tous trois gouverneurs de l'A.O.F.

49. - Pourtant héritier de Léopold Sédar Senghor à la tête du Sénégal (1981-2000), le président Abdou Diouf décide sur ce point de s'éloigner de sa politique de continuité francophile, au moins en ce qui concerne la question de ces symboles très visibles du patrimoine colonial.

50. - « Décolonisation culturelle ». Afrique histoire, 1983, n 9, p. 63.

51. - C'est Hamady Bocoum, chercheur, archéologue de formation et actuel Directeur du Patrimoine culturel du Sénégal, qui a géré à l'époque cette réinstallation. Voir BOCOUM, Hamady. «La protection de l'architecture coloniale au Sénégal : quels enjeux pour le patrimoine? ? Dans Architecture coloniale et patrimoine, tome 1, L'expérience française, direction scientifique de PABOIS, Marc et TOULIER, Bernard. Paris : INP / Somogy - Editions d'Art, 2005.

52. - Le président Abdoulaye Wade est lui-même fils de tirailleur.

53. - Extrait d'un entretien du président sénégalais Abdoulaye Wade avec Michel Lefebvre et Olivier Herviaux, journalistes du Monde paru pour la première fois en novembre 2008, voir le site : http://www.lemonde.fr/societe/article/2013/11/20/abdoulaye-wade-assumer-l-histoire-quellequ-elle-soit_3517273_3224.html (consulté le 24 octobre 2014).

54. - Afin de ne pas susciter de convoitise, nous avons fait le choix de ne pas révéler leur localisation précise.

\section{ABSTRACTS}

The plaster-casts sculpted by Paul Landowski in preparation for his Monument à la Victoire et à la Paix, commemorating the collaboration between French and Moroccan troops during the First World War, have recently been found at Casablanca. But they are not well protected and are threatened with destruction. The work bears witness to the art of the sculptor Landowksi but also to the political issues at stake in the relations between France and Morocco, both during the war and under the Protectorate. The statue was originally erected in the main square of Casablanca, but its transfer in 1961 to Senlis, in France, suggests something of the highly symbolic nature of this cenotaph. This is underlined by the accompanying texts which celebrate the heroism of Moroccan troops not only in the trenches in France, but also, subsequently, in the 'pacification' policies undertaken in Morocco by Lyautey.

Les plâtres sculptés par l'artiste Paul Landowski pour son Monument à la Victoire et à la Paix, commémorant l'entraide entre les troupes françaises et marocaines lors de la Première Guerre mondiale ont été récemment découverts à Casablanca. Ils sont cependant menacés de disparition ou de destruction car ils ne sont pas protégés. L'œuvre du sculpteur témoigne de l'art de Landowski mais aussi des enjeux politiques de la relation entre la France et le Maroc à l'époque du conflit et pendant le Protectorat : en effet, son installation sur la place centrale de Casablanca 
en 1924, puis son retour en France, à Senlis, en 1961, donnent à ce cénotaphe une valeur hautement symbolique, d'autant qu'il est accompagné de textes célébrant aussi bien l'héroïsme des troupes dans les tranchées françaises que leur participation à la politique de "pacification » menée par le résident général Lyautey au Maroc.

\section{INDEX}

Keywords: Landowski, Ducuing, Morocco, Casablanca, Algiers, Dakar, Lyautey, statue, colonies, Senlis, bas-relief, spahi, 'poilu', skirmisher

Mots-clés: Landowski, Ducuing, Maroc, Casablanca, Alger, Dakar, Lyautey, statue, colonies, Senlis, bas-relief, spahi, poilu, tirailleur, monument aux morts

\section{AUTHORS}

\section{NICOLAS ALEXANDRE}

Professeur agrégé d'histoire géographie au lycée Lyautey à Casablanca, Maroc nalexandre@lyceelyautey.org

\section{EMMANUEL NEIGER}

Professeur agrégé de lettres classiques au lycée Lyautey à Casablanca, Maroc, membre de l'association Casamémoire, «Association de sauvegarde du patrimoine architectural du XXème siècle au Maroc » manuneiger@hotmail.com 